\title{
心理咨询理论与实践
}

2019 年 3 月第 1 卷第 1 期

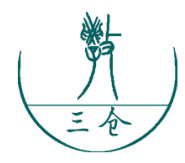

sciscan

\section{台湾青少年女性遭受父亲乱伦行 为的刑案调查}

一以台湾监狱受刑人为研究对象

\author{
蔡政忠 $^{1 *}$ 黄 静 $^{2}$ 黎敏仪 ${ }^{3}$ \\ 1. 福建莆田学院管理学院医疗健康产业管理专业, 福建莆田, 351100; \\ 2. 广州市仲恺农业工程学院人文与社会科学学院, 广东广州, 510550; \\ 3. 澳门大学社会科学学院, 澳门
}

邮箱: 1244528366@qq.com

摘要: 本研究选取台湾地区的台中、彰化、台南、屏东等监狱共计 30 位男 性受刑人作为研究对象, 他们所犯罪行都属于强制性交与猥㗒罪, 而受害人均 为与受刑人有血缘关系之亲生女儿。本文运用定性分析方法并结合文献与访问 调查法收集了《台湾治安事件统计年报》十年来对乱伦案件的分析统计, 得出 受害者有着数种共同的典型特征, 并发现父女乱伦是所有性侵害案件当中最隐 密、最鲜为人知的犯罪。研究采取现象学研究方法, 旨在研究台湾乱伦父亲群 体的犯案过程与其婚姻状况的关系。并分析父亲在进行父女乱伦行为时的心理 认知, 开启华人地区对父女乱伦行为的本土化研究, 将华人父女乱伦犯罪的动机、 成因与社会环境状况提供给教育、社会工作与司法部门，以期为性犯罪防治的 
提供参考依据。

关键词：父女；乱伦；性侵；犯罪；认知扭曲

收稿日期：2018-04-23; 录用日期：2018-05-15; 发表日期：2019-03-15

\title{
Young Women in Taiwan are Under Criminal Investigation of Their Father's Incest_- Study on the Prisoners of Taiwan Prison
}

\author{
Tsai Chengchung $^{1} * \quad$ Huang Jing $^{2} \quad$ Li Minyi $^{3}$ \\ 1.Department of Medical Management, College of Management, Putian 351100; \\ 2.Faculty of Humanities and Social Sciences, Zhongkai University of Agriculture and \\ Engineering, Guangzhou 510225; \\ 3.Faculty of Social Sciences, University of Macau, Macau
}

Abstract: This paper selected Taichung, Changhua, Tainan, Pingdong and other prisons in Taiwan, a total of 30 male inmates as research objects (All their crimes are compulsory and obscene, while the victims are their own daughters ), using the phenomenological method of qualitative research and combined with in-depth interview research methods. It is concluded that the victim has the typical features of several kinds of common, and found that father-daughter incest is the most hidden and least known crime in all sexual assault cases, and analyzes the relationship between the crimes of incest fathers in Taiwan and their marital status, as well as the psychological cognition of father and daughter incest, and the motive of incest crime. The motives, causes and social conditions of incest crime of the father and daughter 
are provided to the education, social work and judicial departments and provide reference for the prevention and control of sexual crimes.

Key words: Father and Daughter; Incest; Sexual Assault; Crime; Cognitive Distortion

Received: 2018-04-23; Accepted: 2018-05-15; Published: 2019-03-15

Copyright (C) 2019 by author(s) and SciScan Publishing Limited

This work is licensed under a Creative Commons Attribution 4.0 International License. https://creativecommons.org/licenses/by/4.0/

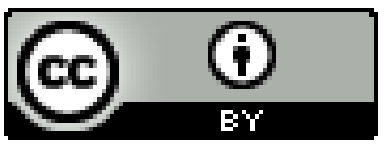

\section{1 背景}

性侵害会造成受害者一生都难以抹灭的记忆，如果再加上乱伦经验则更 容易造成受害者终生难以启齿的伤痛。经历过强暴事件后，受害者一般会回 归家庭来寻求避风港与心理的疗愈，但如果面对的是家中经济支柱且最亲近 的亲人实施的性侵害，这将对受害者本身家庭与社会亲属关系的元认知造成 毁灭性的破坏，受害者未来人生境遇的发展将是后续长久追踪的课题。

Herman（1997）认为乱伦受害者比一般性侵害受害者有着更严重的 PTSD 现象 $[1]$ 。Roth 发现性虐待受害者在成年后有毒品滥用、酗酒、自杀、杀人等 偏差行为的比例高于一般人 [2]；Briere 和 Runtz（1993）对 118 位受到性侵害 女性的研究发现，将近五成是乱伦受害者，其中有七成是在青少年时期第一次 遭遇乱伦事件 [3]。笔者收集了 2000-2010 年近十年来台湾社会乱伦新闻案件, 通过统计分析得出受害者有下列共同特征：（1 ）受害者年龄一般介于 7-12 岁。

（2）遭受乱伦性侵害的受害时间往往超过三年以上，而且多在 14-15 岁才被 
发现。这跟海德瑞特 ( Heitritter) 和弗杰特( Vought) 在 1990年的研究中指出的 “通 常乱伦事件的发生都不会只发生一次，而是持续三到五年的时间”不谋而合。

\section{2 乱伦已报案数与实际案发数的比较}

根据台湾性侵害案件主管机关一一家庭暴力及性侵害防治委员会 2004 年至 2009 年 12 月的统计显示，六年来遭受性侵害犯罪的通报累计已达 46846 件，且 呈逐年递增趋势。其中加害人与受害人属亲属关系的统计案件则有 3561 件，占 总性侵害案件的 $7.6 \%$ 。

根据台湾犯罪学者黄富源（1988）的研究，强奸犯罪的犯罪黑数一般在实 际报案的 10 倍以上, 在所有犯罪类型中隐藏黑数最高 $[4]$; 根据可洁尔 ( Kercher ) 和隆恩（Long）（1991）的粗估，一个性犯罪者每犯下 60 件性侵害案件只会有 一件遭警方逮捕 $[5]$ 。而乱伦案件又属黑数中的黑数, 根据罗雪尔（Russell） 分析仅有 $2.2 \%$ 的家庭乱伦案件曾经报过案，其主因不外 “家丑不可外扬”、年 幼的受害者无法得到其他家人协助报案以及经济上长年依赖加害亲属等 $[6]$ 。

\section{3 引发父女乱伦的各因素分析}

在台湾的统计数据中, 乱伦父亲的年龄一般在 35 至 45 岁之间, 以离婚单 亲家庭并拥有女儿监护权为主要群体, 其中年龄介于 11 到 16 岁、正步人青春 叛逆期的女儿为隐性危险群体。加上女儿在外交到坏朋友、有性滥交与堕胎的 前置条件, 多次遭受家庭暴力才会发生乱伦事件。学者吕琼华将父亲失业列为 引发乱伦行为的前因之一, 笔者研究 30 个个案发现失业因素有 27 件, 占总个 案数的 $90 \%$, 同时乱伦前有酗酒行为者也高达九成; 家中无人，与女儿单独相 处下发生乱伦更是高达十成; 因乱伦遭受惩罚人狱服刑后的再犯比例也是总性 侵害类别中最低的。根据个案汇整发现, 在夫妻未离婚的家庭中发生乱伦案件 主要有下列几种特征：（1）妻子角色功能失调, 妻子对丈夫置之不理以致其性 需求无法满足; (2) 母亲角色功能失调, 没能够尽到照顾女儿的责任, 反而要 求女儿代替母亲照顾弟妹与父亲。

根据家庭功失调观点（Family Dysfunction），乱伦起因是母女家庭角色的互 
换，女儿除照顾弟妹外甚至还需满足父亲精神与身体上的需求。Mclntyre（1981） 指出, 乱伦原因主要在于母亲对妻子与母亲的角色扮演不满, 不愿意成为家人 的支柱与丈夫的性伴侣，并期待家人取代她的角色，甚至把性责任转移至女儿 身上 [7]。但根据笔者的研究发现, 国外家庭功能失调模式并不能完全代表台湾, 因为台湾受到传统儒家五伦观念根深蒂固的影响，若真有不愿意扮演妻子与母 亲角色而发生失调的情况都以夫妻离婚居多，加上台湾的风土民情，法院离婚 案中把女儿监护权判给父亲的案例不在多数。此外, 功能失调论把妻子的软弱、 依赖或经常不在家作为导致乱伦案件产生的原因，这在台湾是不太可能发生的。

\section{4 父亲乱伦行为前后的个人认知}

Barnard 等 (1989) 认为性犯罪的认知可被识别为典型的认知扭曲，从而让性 犯罪者否认、缩小化、合理化其罪行 [8]。Hartley（1998）在分析 8 位乱伦者的 认知类型后认为乱伦犯去除内心对犯罪的压抑认知分别为 “与社会文化相关” “允 许犯案” “削弱责认感” 和 “克服害怕被揭发” 等 4 项 [9]。

\section{1 在犯下乱伦行为前父亲的自我认知}

父亲起先以何种认知方式来说服自己跨越乱伦这道大不韪的门槛，笔者收 集警方 85 份性侵害笔录及 30 位乱伦加害者在法院的开庭供词发现，父亲犯下 乱伦行为前的种种自我认知有以下共同特征：（1）女儿自暴自弃或出现同意的 暗示。个案多数起因于女儿在外行为不检点，如卖淫、援交、滥交、吸毒而导 致离家出走或邽胎等, 父亲在盛怒之下失去理智对女儿进行性侵害。（2）女儿 的行为引诱父亲犯罪。父亲把乱伦动机归各于女儿在家衣衫不整，加上案发前 过度饮酒所致，有些个案因家庭生活贫困、空间狭窄，女儿自幼就跟父母睡在 大通铺或浴室门窗不够隐密让父亲引发遐想而有了可乘之机。（3）食髓知味持 续犯案。乱伦事发后，女儿的行为一如往昔，并未揭发或出现叛逆的行为，让 父亲误认为女儿同意乱伦行为，造成悲剧一再重复上演。笔者研究发现父亲持 续犯罪的另一因素是对女儿恩威并济的冷热态度，除乱伦后加重金钱或亲情的 付出来减轻自身的罪恶感外，另一方面女儿感受到父母离异，父亲飞然一身的 
孤独苦闷而出现 “暂代母职” 的错误想法, 加上有些受害者没能有独立谋生能力, 因此达成另类的不伦交易。

\section{2 乱伦者在案发前如何除去抑制犯罪的做法}

根据上述父亲在进行乱伦行为前错误的自我认知，可归纳出乱伦者在案发 前如何除去抑制犯罪的做法有下列 4 个方面 :

\subsection{1 隐藏在社会文化认知下犯罪}

一般人认为酒后或吸毒容易引发犯罪，因此乱伦者假借酒后对子女进行性 侵犯, 即使当时未有饮酒或吸毒的情况, 但为了躲避刑法的加重刑责, 在坦承 犯案时都会以此为借口。而另外一种形式是假借亲近子女寻求天伦之乐为借口 来侵犯子女, 如猥洃等。

\subsection{2 允诺犯案的认知}

乱伦者会用许多方式来试探子女，如果当时子女没有强烈拒绝，他们会自 我解释为子女以暗示的方式来引诱对其进行性行为, 事后孩子若没有激烈的情 绪表现，乱伦者将会持续犯罪。例如女儿在外发生援交、滥交与堕胎等不良行 为时, 父亲先会以言语刺激试探, 如果孩子表现出只要有钱任何事都愿意的态度, 父亲会以此为借口进行性侵害。

\section{2 .3 减轻罪恶感的认知}

乱伦者会使用不同的方式来减轻对犯罪行为的自责，例如他们会说服女儿 因为父母离婚, 自己有性的需要, 要求女儿暂代妻子职务, 事后以金钱对女儿 进行补偿, 或以亲情来对女儿进行乱伦合理化的说服与洗脑。笔者研究个案时 发现有个乱伦者是高级知识分子, 在乱伦发生后曾搜集文化人类学相关书籍, 并汇总成册，以世界某少数民族对准备出嫁的女儿必须由族中长老进行开苞破 处的仪式来说服女儿。另外在乱伦者供词中普遍声称在犯罪时并未使用暴力来 控制孩子。

\subsection{4 克服被揭发的认知}

众多个案显示, 乱伦者为了避免乱伦行为被揭发, 会处心积虑地利用女儿 在外交到坏朋友等方式来躲避被揭发的恐惧, 他们认为即使罪行被揭发, 女儿 
周遭亲戚朋友也不会相信或提出诉讼。

\section{3 案发当时乱伦父亲看待受害者的错误认知}

根据笔者研究汇整警方 30 位乱伦父亲的笔录发现，在案发当时乱伦父亲看 待受害者的错误认知可分为以下 4 种:

\section{3 .1 妻子与女儿身份的误判}

乱伦多起因于平日的酗酒行为，加上酒后乱性，把女儿错看成妻子的情况 在警方笔录中出现最多，加上乱伦者与妻子错综复杂的感情纠葛让女儿成了父 母婚姻的受害者。

\subsection{2 性发泄对象}

长期分居、丧偶或离婚，加上失业、负债等经济压力，让若困的父亲无法 有足够的金钱在外寻欢作乐，进而把对感情及性的需求移转到受监护子女身上。 调查发现，乱伦父亲除了把女儿当成性发泄的对象之外，也或多或少地将女儿 当成妻子的角色，不只是单纯性发泄，而且混杂了一种对妻子和对女儿的 “如 父如夫” 的复杂情感，后续对乱伦父亲的情感追踪发现，这种情感有异于他们 在外买春的心态。

\subsection{3 对女儿的错误期望}

除了上述将女儿误认为妻子加以性侵犯之外，个案研究发现因离婚、丧偶 等因素让乱伦父亲认为世事无常，现今只剩父女俩相依为命，而产生不想改变 当前仅存少数薄弱的亲属关系的想法，这份孤寂感让乱伦父亲产生不希望女儿 嫁人的占有欲望，进而加以性侵犯。

\subsection{4 女儿身份的缩小化}

父亲以女儿在外滥交、援交、卖淫、堕胎、吸毒等不良行为，试图建立女 儿并非单纯无辜的形象，以达到受害者身份的缩小化。有些乱伦父亲误认为女 儿之所以交坏朋友的主要原因是对性的好奇与需求，为了想留住女儿，不在外 游荡等，以管教为借口而主动对女儿提供性的满足与慰藉，甚至于有些个案显 示女儿还会主动地向父亲要求性交，然而研究者发现此类动机是乱伦性交后父 亲提供可观的财物以满足女儿对奢侈品的需求。 


\section{5 父亲乱伦行为遭受司法审判时的认知与态度}

根据笔者调阅法院 115 份与乱伦有关的法庭审判纪录以及 30 份与本研究有 关的法院判决书发现，在法官面前嫌犯对乱伦事件坦承犯案的态度，一般为以 下 3 种:

\section{1 消极否认}

虽然承认乱伦但对乱伦当时的前因后果的细节均未坦白交待，以“时间久 远忘记了”“当时喝了酒记不清楚” “好像有又好像没有” 等语气含糊带过。

\section{2 栽赃受害型的积极否认}

乱伦嫌犯在法庭上对犯罪行为表示出完全否认的态度, 并提出明确具体事 证向法官表示女儿因长期对自己管教方式不满而心生报复，或指出女儿另有图 谋并与外人勾结设下陷阱想在自己身上获取利益或监护权等。该情况经常涉及 夫妻情感破裂后的监护权之争，或因子女吸毒、奢博惹下庞大债务，或凯鄃见父 亲名下资产设计陷害等，情节复杂。

\section{3 缄默型的承认犯罪}

本研究的 30 位已人狱服刑的乱伦犯，在事后对其进行访谈，在回答 “法院判 决二到十年不等的乱伦罪还有哪些想法” 时，其一般呈现出 “辩解会让女儿再度 遭受伤害” “我含冤承认的目的是为了让女儿回头，改过向善” “这社会就是这 样，小孩说一句，大人再怎么辩解都没用” “我只犯过一次，她却反咬好几次， 甚至说好几年，但最后一样要被关，只好随便她讲” 等等的无奈。值得注意的是， 对于乱伦罪行法院判决书中记载内容与笔者对当事者进行罪行陈述与访谈的细节 有颇多出人，但共通的一点是在司法刑事庭时，所有犯罪者都承认乱伦罪行。

笔者研究父亲的乱伦行为遭法院起诉并在律师的陪同下辩护时发现，犯罪 者与委任律师之间在消弭或闪避加害人法律与良心谴责时出现下列共同特征:

\subsection{1 否认使用暴力}

对警方的笔录汇整后发现，乱伦父亲一致否认对女儿使用暴力。笔者与检 
警交流后得知乱伦者之所以否认使用暴力是因为想躲避加重伤害等相关刑事责 任。至于案发当时父亲是否使用暴力则见人见智。根据前述对乱伦行为认知的 资料汇整发现，所有乱伦父亲均不承认对女儿使用暴力且在事后以金钱或女儿 想要的物品来进行减少罪恶感的另类交易。令人怀疑的是为何所有受害者的警 方供词都出人意料地一致出现女儿控诉父亲使用暴力，而这项说词居然会一致 让法官采信？笔者调阅法庭记录的内容，虽然乱伦者们对此指控都保持缄默， 但笔者进行访谈时却都否认有乱伦前施暴的种种行为。笔者与乱伦者讨论到该 案情况时, 他们认为会造成父女乱伦本来两方都有错, 但女儿本身行为不检点 在先，加上在外面交到坏朋友想回家找父亲勒索财物不成而想出种种陷害父亲 的方法。有些父亲只有猥玟行为却硬被说成乱伦的性行为, 有些只有发生一两次, 因良心发现而拒绝再犯，却被女儿说成性侵犯多年。但父亲却为何在法庭保持 缄默不予反驳的主因除了不想对女儿造成二度伤害之外，还想牺牲自己来独自 承受不实指控以换取女儿良心发现，浪子回头。

\subsection{2 向女儿坦白性需求}

因婚姻的破裂父女相依为命的情况之下，父亲在初次性侵犯女儿后，女儿 态度并未表示明显反感且一如继往地与父亲保持亲子互动，而食髓知味的父亲 每次都会向女儿表示自己有性需求。根据统计资料显示，该类型的父亲呈现出 异于酒后乱伦的较为理性的态度，但因性需求无处发泄又明知与女儿乱伦有违 道德，故在性交前都会对女儿表示歉意并把乱伦行为归处于女儿母亲的抛夫弃 子行为，为人子女此时此刻更应该 “子代妻职” ，乱伦后也会以提供财物等方 式进行补偿。

\subsection{3 动机良善论}

针对上述案例显示，乱伦父亲认为女儿在外行为不检点、援交、滥交或长期 在外游荡，自以为可以用性行为来满足女儿对异性的需求，才会发生乱伦事件， 例如 “只要她不在外头搞七捻三，我就算下地狱也无妨” “她想认识异性，我出 发点在管教，没有错，但我用错了方法”。 


\section{6 如何抑制乱伦犯罪的几点建议}

\section{1 亲子教育的监护与强化}

除了学校应在课程中加强婚姻与亲子教育的观念之外，学校社工和班主任 应防患于未然，一旦发现有家庭暴力, 就应该积极介人处理, 并对学生心理或 行为的异常进行辅导。

\section{2 通畅儿童与青少年性侵害的“学校社工”通报机制}

儿童或青少年遭到性侵害时，一般难以启齿，受害者顶多只向较为亲近的 同学诉苦, 因此学校应该研究如何做好师生多元信息通报渠道, 并结合学校心理 辅导中心与地区警方派出所等做好危机处理工作，在秘而不宣保护受害学生的前 提下, 对性侵害本身性质的禁忌与隐讳性及加害人要胁受害人不能报案等心态进 行合理处理。无论何种情况，儿童受害人甚至其家人都可能需要社工人员扶助、 安抚情绪、陪同验伤采证、协助取得各种社会资源、解释及陪伴面对的司法过程 和陪同出庭。陪伴本身即是一种心理支持, 社工人员的陪伴尤其代表了社会在司 法正义之外的弥补。儿童受害人心理的创伤, 较成人更为严重, 造成创伤后压力 症候群的几率更高, 以后产生忧郁症、自杀、药毒瘾、酒瘾、暴力犯罪等问题的 几率上升, 所以必须照会精神科医师对其做必要的评估及药物或心理治疗, 以减 少身体、精神、人格及行为上种种并发问题的可能性。

\section{3 法院对子女监护权的判决}

众多个案一致发现，乱伦均发生于单亲家庭的父女，加上父亲经济状况与 收人不稳定, 致使乱伦发生几率大增。未来司法机关在对子女监护权进行判决时, 可以参考父亲与女儿的相对年龄来进行监护权的归属判决, 若父亲年龄与未成 年女儿的年龄差距在本研究的危险范围之内, 加上父亲无意愿结交新的异性伴 侣以及考虑到少女初次生理知识的教育等问题, 法官监护权判决应以女儿人身 安全为首要考量。 


\section{4 善用传媒宣导性犯罪的处遇方式}

适当地使用媒体这一在当今社会具有强大影响力的渠道来提升公众认知， 进行大范围的教育宣传。从而让儿童性侵犯这一人人避而不谈的私密话题，成 功转人公共视角, 为广大人民群众所讨论, 这一举措对打破中国社会根深蒂固, 甚至为大家所默认的 “沉默文化” 具有极大的影响。例如，由曾经有媒体发起 形成的公益组织 “女童保护” 在三年内无间断地进行调研并将其结果公之于众, 着重对儿童性侵害现象的重要性进行强调, 统计数据中也包含了发生率的变化。 中国著名律师佟丽华所在的援助中心在曾经向全国妇联出具过调研报告，这份 报告整合了见于媒体报道的儿童性侵案件，累积已经达到 800 多件，“作案人 和受害者的特质没有发生多大的改名，熟人作案这一比例依然在七成左右”。 一般性侵害对象为女性与未成年人，在遭受性侵犯事件之后普遍没有危机处理 知识, 进而丧失举证良机与严惩加害人为自己主持法律公道的机会, 为此大众 传媒不仅要扮演好性别学习的社会角色，在制作节目时也应该传达法律与社会 救助等知识, 透过媒体影响来扭转民众看待性侵犯或乱伦事件 “难以启齿” 或 “家 丑不可外扬” 的驼鸟心态，让亲友、师生和社会都能成为性侵犯受害者强有力 的身心依靠。

\section{5 提升社工及医疗人员专业水平}

为提升对儿童性侵害被害人的验伤采证及医疗服务质量，相关专业医疗人员 应持续接受临床法医学的验伤采证训练，把儿童性侵害个案集中于特定验伤中心 检伤、追踪辅导及提供精神治疗，且建立专家共同审查案件及跨专业定期讨论个 案的制度, 才能累积经验、加强服务、继续教育及持续改善。在司法单位核可之下， 让有心理、语言、儿童发展及相关法律等专业训练的人，在儿童感觉舒适且不受 干扰的环境中，用儿童能理解且规范的问题模式，在没有诱导性用语及污染证词 的疑虑之下，使儿童能陈述事件发生的经过及相关情况。这样的专业人员，在美 国称为 “Forensic interviewer”。“Forensic” 是法律的意思，“interviewer” 是面 谈及询问的人员，结合起来是为司法目的作面谈询问的人员。Forensic interviewer 是在美国开始发展的专业，目前已推广至英国及欧洲各国，甚至在日本及韩国也 
已经开始进行训练。由 Forensic interviewer 所做的记录，可以得到法庭信任，以 传闻证据视之，作为呈堂证据，避免污染证词，减少重复陈述，尤其能为儿童及 心智障碍等弱势被害人提供司法服务，达到巩固供述证据的目的。

\section{参考文献}

[ 1 ] Herman J. Trauma \& Recovery [ M ] . New York: Basic Books, 1997.

[2] Roth S, Nerman E.The process of coping with incest for adult survivors: measurement and implications for treatment and research $[\mathrm{J}]$. Journal of Interpersonal Violence, 1993 ( 8 ) : 363-377.

[3] Briere Z, Runtz M.Childhood sexual abuse: Long-term sequelae and implication for psychological assessment.Journal of Interpersonal Violence, 1993 ( 8 ) : 312-330.

[4] 黄富源, 黄征男. 性侵害加害人之特质与犯罪手法之研究 [ M ] . 台北: 三 民书局，1999.

[5] Quinsey V L, Lalumiere M L, Rice M E, et al.Predicting sexual offenders [ M ] . Campbell J C.Assessing dangerousness: Violence by sexual offender, batterers, and child abusers.Thousand Oaks, CA: sage, 1991: 114-137.

[6 ] Russell D E H.The secret trauma-incest in the lives of girls \& women [ M ] . New York: Basic Book, Inc., 1986.

[ 7 ] Mclntyre K.Role of mothers in father-daughter incest: A feminist analysis [ J ] . National Association of Social Worker, 1981: 462-466.

[8] Barriga A, Gibbs J.Measuring cognitive distortion in antisocial youth: Preliminary Development and validation of the How I Think Questionnaire $[\mathrm{J}]$. Journal of Aggressive Behavior, 1996 ( 22 ) : 333-343.

[9] Hartley C C.How incest offenders overcome internal inhibitions through the use of cognitions and cognitive distortions $[\mathrm{J}]$. Journal of Interpersonal Violence, 1998, 13 ( 1 ) : 25-40. 